\title{
The effect of mastectomy and radiotherapy for breast carcinoma on soft tissues of the shoulder and its joint mobility among Egyptian patients
}

\author{
G.M. SAIED ${ }^{1}$, R.M. KAMEL ${ }^{2}$ and N.R. DESSOUKI ${ }^{*}$ \\ ${ }^{1}$ Department of General Surgery, Faculty of Medicine, Cairo University, Egypt \\ ${ }^{2}$ Department of Basic Sciences, Faculty of Physiotherapy, Cairo University, Cairo, Egypt \\ ${ }^{1}$ Department of General Surgery, St. Bernard's Hospital, Gibraltar
}

\begin{abstract}
Patients with post mastectomy soft tissue shoulder disorders usually benefit from various lines of physiotherapy treatment. However, the controversy about their efficacy persists. The aim of this work was to study and assess the efficacy of each, and to identify the best intervention. One hundred female patients with ipsilateral post mastectomy shoulder problems were enrolled in the study, from September 2003 until December 2004. They were followed up for 32 weeks. Mastectomy, both radical and conservative and axillary lymph node clearance, was the standard surgery applied for operable breast carcinoma in this series. Clinical examination was followed by testing for the shoulder complaint by measuring maximal protrusion at the inferior scapular angle, scapular stabilization and the lift-off tests. Approved physiotherapy modalities were then applied, viz: no treatment (randomly chosen 12 patients), passive and active motion therapy (14 patients), oral diclofenac sodium (19 patients), local triamcinilone injection (40 patients) and manually applied low intensity laser therapy (15 patients). Assessment was by determining overall success rate for each intervention modality. Intervention outcome was assessed at 8,16 , and 32 weeks as shown by physical examination using the healthy shoulder as a reference, and by measuring restricted mobility during passive lateral rotation and glenohumeral abduction. "Success rate" was determined separately for each group at the end of the intervention period. The applied surgery was followed by radiotherapy in $96 \%$, chemotherapy in $24 \%$ and both in $11 \%$. The presenting post mastectomy symptoms at the shoulder were pain (100\%), shoulder weakness $(88 \%)$, winging of the scapula $(11 \%)$ and inability to perform everyday shoulder movements $(23 \%)$. Evaluation was by overall improvement score. The results were: $14.3 \%$ for untreated patients, $43.3 \%$ for those treated by motion therapy, $42 \%$ for diclofenac therapy, $80.7 \%$ for local triamcinolone, and lastly $68 \%$ for low intensity laser therapy. All treatment regimens for shoulder disabilities in those patients gave little long-term advantage, local steroid injections were the most effective. Low level laser therapy may augment its effect. It is concluded that all treatment regimens provide little long-term advantage; however, trimcinilone local injections may be the most useful in terms of pain relief and improvement in shoulder movement.
\end{abstract}

Key words: shoulder, disorders, mastectomy, radiotherapy, breast, cancer, Egypt

\section{Introduction}

Like many other parts of the world, carcinoma of the breast is the commonest female cancer in Egypt. It maximally occurs in the $4^{\text {th }}$ decade of life and causes the greatest number of cancer deaths in females. Risk factors include family history of breast cancer, long child bearing period, nulliparity, and a rich fat diet (McArdle et al. 2000). The surgical treatment for breast cancer in Egypt is simple mastectomy and breast conserving surgery (wide local excision, quadrantectomy or segmentectomy). Adjuvant therapy includes radiotherapy, chemotherapy and hormonal treatment. Seventy per cent of cancer patients experience loss of energy and impairment of physical performance during their illness (Dimeo et al., 1997). Recumbence in bed may also lead to muscular weakness and reduces working hours (Taylor et al., 1949).
The treatment of breast cancer itself may be responsible for neuromuscular complications. The previously reported brachial plexus injury is now rare (Bland \& Copland, 1991). Winging of the scapula due to injury of the long thoracic nerve, is uncommonly reported and represents a significant functional problem with limitation of shoulder elevation and flexion (Waiter \& Flatow, 1999; Sounders, 1995). The diagnosis of long thoracic nerve injury is possible by clinical examination. In addition, bedside tests help to confirm and include measuring the maximal protrusion at the inferior scapular angle in centimetres and scapular stabilization test(Waiter \& Flatow, 1999). Long thoracic nerve conduction study electromyography (EMG) and magnetic resonance imaging (MRI) scans may also be of value. EMG is helpful to confirm if continuity exists or not (Iceton \& Harris, 1987). Injury to nerve to subscapularis is recognized late and

“Correspondence: Nazar R. Dessouki; E-mail: nazar_dessouki@doctors.org.uk 
causes loss of internal rotation strength giving a negative 'lift off' test (Clark et al., 2002). This test is important as the muscle is not accessible to observation or palpation.

Decreased shoulder mobility (frozen shoulder) due to capsulitis is a serious clinical finding, particularly if accompanied by decrease in shoulder range of motion. If present it is probably caused by irradiation injury. Spontaneous improvement of serratus anterior weakness may occur, and it is important to try to maintain full range of movement with exercises, and prevent contracture until this happens (Warner \& Navarro, 1998). Early range of motion therapy to the shoulder (with adequate analgesia and muscle relaxants) is needed for treating other disabilities irrespective of the cause. It reduces muscle spasm while maintaining full range of motion. Vigorous and forceful exercises are contraindicated because of the pain associated with the rupture of adhesions. Nonsteroidal anti-inflammatory drugs and local corticosteroid injections help to relieve pain and inflammation. Late disease requires manipulation under anaesthesia to sever the adhesions. Strengthening of the remaining functioning muscles will help to maintain shoulder integrity. The average recovery time is nine months and treatment should be only considered after two years without evidence of nerve recovery (Connor et al., 1997; Kauppila \& Vastamaki, 1996). Surgical decompression and neurolysis of the long thoracic nerve is sometimes needed, while orthopaedic procedures as scapulothoracic fusions, static stabilization procedures, and dynamic muscle transfers, are rarely considered (Bunch \& Siegel, 1993; Hawkins et al., 1990).

The objective of this work was to study neuromuscular disorders at shoulder region in Egyptian females having ipsilateral breast carcinoma, and to evaluate complications of adjuvant therapies on the region.

\section{Materials and Methods}

Giving an informed consent, 100 patients treated of breast cancer (including 12 controls), having shoulder pain and restricted mobility, were enrolled in this study. They presented three months after the completion of treatment, and were followed up for eight months. Clinical examination for the shoulder disability included patient criteria, type of treatment applied, period of postoperative status and neuromuscular symptoms at the shoulder. Clinical testing for the shoulder complaint included "maximal protrusion at the inferior scapular angle test" for serratus anterior strength, "scapular stabilization test" and the "lift-off test" which isolates subscapularis injury (Clark et al., 2002).

The treatment modalities applied included (i) a motion therapy (14 patients) whereby assistive passive exercise was performed for the patient and resistive active exercise done by the patient (e.g. Codman exercise, push-ups, and chinning bar) (Cailliet, 1979); (ii) diclofenac sodium as nonsteroidal anti-inflammatory drug combined with the centrally acting skeletal muscle relaxant dantrolene to potentiate action (19 patients). Two of the patients had had previous history of gastric troubles, and $30 \mathrm{mg}$ of the gastroprotective lanzoprazole was added to treatment; (iii) repeated injections of $30 \mathrm{mg}$ of the locally acting steroid triamcinolone acetonide (40 patients) mixed with the local aesthetic lidocaine and injected $1 \mathrm{~cm}$ distal and lateral to the coracoid process (Martz, 2003); (iv) manually applied low intensity infrared laser (15 patients) using a hand piece directed perpendicular to the skin surface, one minute for each selected point (Nykanen, 1995). Laser specifications were wave length $409 \mathrm{~nm}$, average output power $5 \mathrm{mw}$, frequency $5000 \mathrm{~Hz}$, pulsed (200ns), mode and energy density $1 \mathrm{Jule} / \mathrm{cm}^{2}$ and duration 20 minutes; and (v) manipulation under anaesthesia to break up adhesions (for a single patient having serratus anterior paralysis).

The intervention outcome was assessed at 8,16 , and 32 weeks. It included physical examination (using the healthy shoulder as a reference) to measure the restriction of mobility during passive lateral rotation and glenohumeral abduction. The proportion of patients improved in relation to the total (known as the "success rate") was determined separately for each group at the end of the intervention period. Complete recovery or much improvement as judged by the patient was counted as success. Pain was scored on a $100 \mathrm{~mm}$ visual analogue scale; zero being no pain while a score of 100 indicated very severe pain (Carlsson, 1983). 


\section{Results}

One patient withdrew from the study immediately after enrolment, with no clear explanation and was excluded. All patients were females with mean age of 48 years. The lesions and their surgery (with or without adjuvant therapy) were affecting the left breast in $58 \%$ of cases and the right breast in $41 \%$. Bilateral operations - one year apart, were done for a single patient. Radiotherapy combined surgery in $96 \%$, while chemotherapy combined in $24 \%$ and both in $11 \%$. Some patients were receiving other medications like the anti-oestrogen tamoxifen or vitamins. The presenting symptoms for physiotherapist were: pain $(100 \%)$, shoulder weakness $(88 \%)$, winging of the scapula (11\%) and inability to perform every day shoulder movements such as combing and cooking in $23 \%$. All patients received one or more of the programmed treatment modalities, except 12 who represented a control group and received no treatment.

Table 1: Number of treatment, and success rate of different treatment modalities

\begin{tabular}{lrrrrr}
\hline Index $(\%)$ & No. of treatment & Motion therapy & Local trimcnilone & Diclofenac & LILT \\
& $\mathrm{n}=12$ & $\mathrm{n}=14$ & $\mathrm{n}=40$ & $\mathrm{n}=19$ & $\mathrm{n}=15$ \\
\hline Physical examination & 20 & 44 & 82 & 38 & 68 \\
Success rate & 11 & 38 & 72 & 28 & 70 \\
Visual analogue scale & 88 & 52 & 12 & 40 & 34 \\
Overall success rate & 14.3 & 43.3 & 80.7 & 42.3 & 68 \\
\hline
\end{tabular}

Key: LILT= low intensity laser therapy

Assessment was given a percentage value using three different parameters: subjective success as projected by patients, objective success as reported by the physiotherapist (following physical examination) and assessment of pain by visual analogue scale. Each patient had an overall improvement score calculated from these indices. It was: $14.3 \%$ for untreated patients, $43.3 \%$ for motion therapy, $80.7 \%$ for triamcinolone, $42 \%$ for diclofenac and lastly $68 \%$ for laser (Table 1 ).

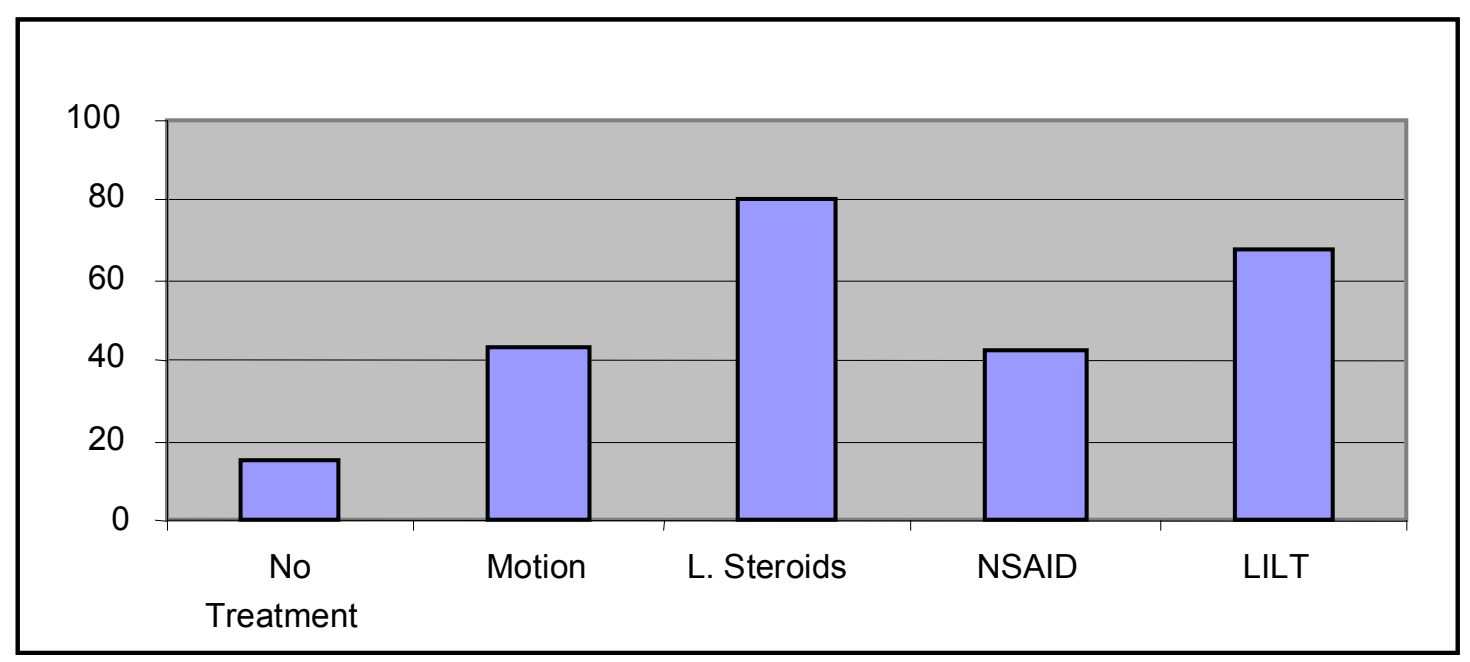

Figure 1: The overall success rate of the treatment modalities 
The overall success rates were high when using local steroids or low intensity laser therapy (Figure 1)

\section{Discussion}

Patients with shoulder disorders after breast cancer treatment are a challenging issue for both surgeons and physiotherapists. Correction of any muscle imbalance is paramount to preservation of mobility and function of the whole upper limb. Preventive rehabilitation before treatment may limit impairments and also decreases the need for supportive and palliative rehabilitation which is usually unsatisfactory.

So far there are no firm conclusions as to the best and most effective treatment protocol. At Albert Einstein Medical Center, the rehabilitation programme included dancing with the aid of an expert dancer, for both physical and psychological benefit, release of tension and decrease of pain (Malenaro et al., 1986). It also provides the patient with an emotional stimulus that emphasizes her femininity and sexuality during her adjustment to a new body image, and helps her to feel "whole again". Though hard to apply in Egypt, in a dancing programme one should concentrate on muscles whose function is affected by the mastectomy. Isolated muscle impairment as the subscapularis is usually treated conservatively and a substantial improvement occurs without surgery. Those patients usually suffer significant night pain and present late, but respond appreciably to physiotherapy.

Research studies demonstrate that ultrasound treatment has very little value, despite its known effects upon living tissues (Berry et al., 1980). This is due to the chronic and degenerative nature of the lesion, and also to individual variations. For this reason it was not included in the present study.

Nonsteroidal anti-inflammatory drugs (NSAID) are routinely used in shoulder disabilities (Almekinders, 1993) as they reduce pain and swelling, and help the patient to perform earlier motion during recovery. But it seems that their effect is overestimated, as they gave only $42 \%$ overall success rate in our series with maximal effect on pain. The contraindications to their use are patients over 60 years old, arthritis, history of peptic ulcer, and concomitant anticoagulant or corticosteroid therapy. In general authors recommended routinely combining them with lanzoprazol, irrespective of age or the presence of any risk factors.

Corticosteroid injections usually give superior results to other forms of treatment, with faster relief of symptoms. In the present series they gave almost double the effect of NSAIDs with maximal benefit on pain relief. The idea behind using triamcinolone acetonide in particular was its medium duration of action compared to hydrocortisone, and is 5 times more potent (Martz, 2003). Adverse reactions are generally mild but one should be aware of them particularly in women. They are contraindicated if there is history of allergy to the drug, presence of nearby infections and in coagulopathy. Diabetes mellitus and anticoagulation therapy are relative contraindications.

Low level laser therapy though reported successful in some studies, yet its effectiveness proved to be very short term if compared to nonsteroidal drugs or to placebo (Nykanen, 1995). Laser therapy augments collagen synthesis, modulates its maturation, and above all enhances the biomechanical characteristics of healing tissues at the shoulder region injured by operation or radiotherapy. This effect is promoted if combined with motion therapy. Due to the relatively high overall success rate in our patients we recommend its use in addition to local steroid injection and ultrasound therapy.

In conclusion, this study has shown that all treatment regimens give little longterm advantage. However, trimcinilone local injections may be the most useful in terms of pain relief and improvement in range of movement in the early stages of the disability. By combination with low level laser therapy, the results may be augmented.

\section{Acknowledgements}

We thank Dr. Azza Saied of the Faculty of Engineering, Cairo University, for partial assistance.

\section{References}

Almekinders, L.C. (1993) Anti-inflammatory treatment of muscular injuries in sports. Sports Medicine 15, 139-145.

Berry, H., Fernandez, L., Bloom, B., Clark, R.J. \& Hamilton, E.B. (1980) Clinical 
study comparing acupuncture, physiotherapy, injection and oral antiinflammatory therapy in shoulder-cuff lesions. Current Medical Research and Opinion 7, 121-126.

Bland, K.I. \& Copland, E.M. (1991) The Breast: Comprehensive Management of Benign and Malignant Diseases. Philadelphia, London, Toronto, Tokyo, W.B Saunders pp.695.

Bunch, W.H. \& Siegel, I.M. (1993) Scapulothoracic arthrodesis in fascioscapulohumeral muscular dystrophy: review of seventeen procedures with three to twenty-one year follow-up. Journal of Bone and Joint Surgery 75A, 372-376.

Cailliet, R. (1979) Shoulder Pain. F.A. Davis Company, Philadelphia. pp 42-57.

Carlsson, A.M. (1983) Aspects of the reliability and validity of the visual analogue scale. Pain 16, 87-101.

Clark, R.J., Marchessault, J., Sizer, P.S. Jr. \& Slauterbeck, J. (2002) Isolated traumatic rupture of the subscapularis tendon. Journal of the American Board of Family Practice 15, 304-308.

Connor, P.M., Yamaguchi, K., Manifold, S.G., Pollock, R.G., Flatow, E.L. \& Bigliani, L.U. (1997) Split pectoralis major transfer for serratus anterior palsy. Clinical Orthopaedics and Related Research 341, 134-142.

Dimeo, C.F., Monika, H.M., Hartmut, B., Loather, K., Roland, M. \& Joseph, K. (1997) Aerobic exercise in the rehabilitation of cancer patients after high dose chemotherapy and autologous peripheral stem cell transplantation. Cancer 79, 1717-1722.

Hawkins, R.J., Willis, R.B. \& Litchfield, R.B. (1990) Scapulothoracic arthrodesis for scapular winging. In: Surgery of the Shoulder (M.
Post, B.F. Morrey \& R.J. Hawkins (eds). St. Louis, Mosby Year Book, 356-359.

Iceton, J. \& Harris, W.R. (1987) Treatment of winged scapula by pectoralis major transfer. Journal of Bone and Joint Surgery 69B, 108-110.

Kauppila, L.I. \& Vastamaki, M. (1996) Iatrogenic serratus anterior paralysis: long-term outcome in 26 patients. Chest 109, 31-34.

Malenaro, J., Kleinfeid, M., Lebed, S. (1986) Physical therapy and dance in the management of breast cancer. Physiotherapy 66, 967-969.

Martz, W.D. (2003) Corticosteroid injections for articular and soft tissue pain. Patient Care 37, 39-48.

McArdle, W.D, Katch, F.I. \& Katch, V.L. (2000) Exercise Physiology Energy, Nutrition and Human Performance. $5^{\text {th }}$ ed. Lippincott Williams \&Wilkins. Pp. 917-922.

Nykanen, M. (1995) Physiotherapy for patients with soft tissue shoulder disorders: a systematic review of randomized clinical trials. Scandinavian Journal of Rehabilitation Medicine 27, 105-108.

Sounders, L. (1995) The efficacy of low level laser therapy in supraspinatus tendinitis. Clinical Rehabilitation 9, 126-34.

Taylor, H.L., Henschel, A., Brozek, J. \& Keys, A. (1949) 'Effects of bed rest on cardiovascular function and work performance.`Journal of Applied Physiology 2, 223-239.

Waiter, J.M. \& Flatow, E.L. (1999) The long thoracic nerve. Clinical Orthopaedics and Related Research 368, 17-27.

Warner, J.J.P. \& Navarro, R.A. (1998) Serratus anterior dysfunction: recognition and treatment. Clinical Orthopedics and Related Research 349, 139-148. 